\title{
Magnetically Driven Floating Foams for the Removal of Oil Contaminants from Water
}

\author{
Paola Calcagnile, ${ }^{\dagger, *}$ Despina Fragouli, ${ }^{\dagger, *}$ Ilker S. Bayer, ${ }^{\dagger}$ George C. Anyfantis, ${ }^{\dagger}$ Luigi Martiradonna, ${ }^{\dagger}$ \\ P. Davide Cozzoli, ${ }^{\neq, \S}$ Roberto Cingolani, ${ }^{\perp}$ and Athanassia Athanassiou ${ }^{t, \perp}$ \\ ${ }^{\dagger}$ Center for Biomolecular Nanotechnologies @UNILE, Istituto Italiano di Tecnologia (IIT), via Barsanti, 73010 Arnesano (LE), Italy, ${ }^{\ddagger}$ National Nanotechnology \\ Laboratory (NNL), CNR - Istituto Nanoscienze, c/o Distretto Tecnologico, via per Arnesano km 5, 73100 Lecce, Italy, ${ }^{\S}$ Dipartimento di Matermatica e Fisica \\ "E. De Giorgi", Università del Salento, via per Arnesano, 73100 Lecce, Italy, and ${ }^{\perp}$ Istituto Italiano di Tecnologia (IIT), via Morego 30, 16163 Genova, Italy
}

$\mathrm{O}$ il and fuel spills released during industrial accidents or oil tankers and ships sinking are catastrophic for the marine and aquatic ecosystems. To avoid such environmental disasters, immediate polluted water purification and long-term water preservation are essential. These issues have inspired the recent scientific research in the field of materials science, with numerous studies aiming at the development of new materials for "water-oil separation". ${ }^{1-6}$ The large-scale application of this research area points toward highly efficient but, at the same time, low-cost systems. A common approach of the previous studies is the combined control of the chemical and structural properties of the developed materials in order to exhibit simultaneous superhydrophobicity and superoleophilicity.

So far, superhydrophobic and superoleophilic meshes, ${ }^{2,5-8}$ films, $^{9}$ or membranes ${ }^{10-13}$ have been used as filters that allow oil to penetrate through them and keep water on their surfaces. Although they can separate oil contaminants from water efficiently, they cannot be applied in the place of the oil spills because the polluted water should be first collected and then be filtered. Alternatively, there are fabricated materials that can selectively absorb oil contaminants from a water-oil interface, with the majority of them presenting various drawbacks which limit their mass applicability. In particular, recent studies present highly hydrophobic and oleophilic magnetic spheres, which can be placed on the polluted water zone and subsequently be removed by means of an external magnetic field (MF). ${ }^{14,15}$ While these studies have the merit of introducing the magnetic actuation, the large quantities of microspheres needed for purification of extensively polluted areas prohibit their large-scale application.
ABSTRACT In this study, we present a novel composite material based on commercially available polyurethane foams functionalized with colloidal superparamagnetic iron oxide nanoparticles and submicrometer polytetrafluoroethylene particles, which can efficiently separate oil from water. Untreated foam surfaces are inherently hydrophobic and oleophobic, but they can be rendered

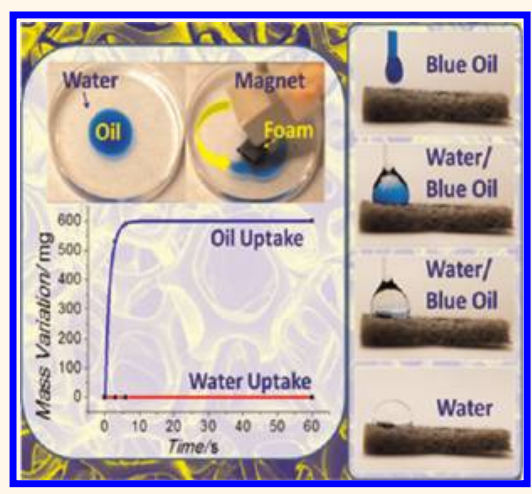
water-repellent and oil-absorbing by a solvent-free, electrostatic polytetrafluoroethylene particle deposition technique. It was found that combined functionalization of the polytetrafluoroethylene-treated foam surfaces with colloidal iron oxide nanoparticles significantly increases the speed of oil absorption. Detailed microscopic and wettability studies reveal that the combined effects of the surface morphology and of the chemistry of the functionalized foams greatly affect the oil-absorption dynamics. In particular, nanoparticle capping molecules are found to play a major role in this mechanism. In addition to the water-repellent and oilabsorbing capabilities, the functionalized foams exhibit also magnetic responsivity. Finally, due to their light weight, they float easily on water. Hence, by simply moving them around oilpolluted waters using a magnet, they can absorb the floating oil from the polluted regions, thereby purifying the water underneath. This low-cost process can easily be scaled up to clean large-area oil spills in water.

KEYWORDS: colloidal nanoparticles - foams - magnetic actuation superhydrophobic $\cdot$ superoleophilic

In addition, recently developed carbon foam systems, although they present high absorption efficiency, are fabricated using expensive materials and sophisticated procedures, ${ }^{16}$ limiting their mass production. A simpler approach is the fabrication of polymeric or nanocomposite foams, as presented in various recent studies. ${ }^{17-19}$ However, in some cases, the wetting properties of the systems are either not characterized ${ }^{17,18}$ or they do not show the desirable superhydrophobicity and superoleophilicity, ${ }^{19}$ resulting in low separation efficiency.

\section{* Address correspondence to paola.calcagnile@iit.it, despina.fragouli@iit.it.}

Received for review March 23, 2012

Published online May 11, 2012 $10.1021 / \mathrm{nn} 3012948$

(c) 2012 American Chemical Society and accepted May 11, 2012. 


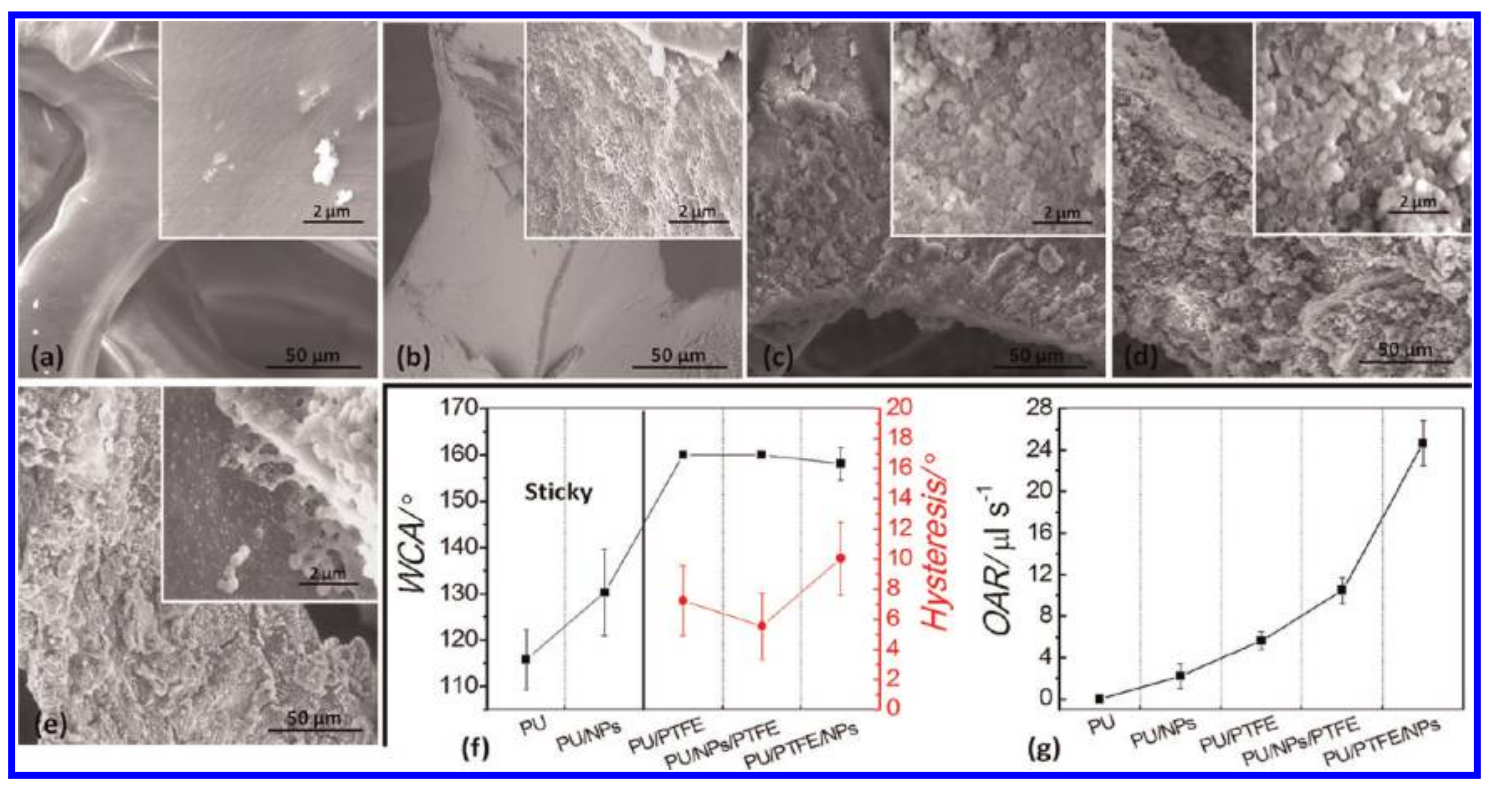

Figure 1. SEM images of (a) PU foam, (b) PU/NPs, (c) PU/PTFE, (d) PU/NPs/PTFE, (e) PU/PTFE/NPs. Insets show higher magnification images. (f) WCA (black squares), water hysteresis (red circles), and (g) OAR measurements, performed on all five samples.

\section{RESULTS AND DISCUSSION}

Here, we obtain a composite magnetic material, with highly efficient oil/water separation capability, using a facile and easily scalable fabrication technique based on inexpensive materials. The starting material is commercially available polyurethane (PU) foam, which is widely used for various everyday life applications such as packaging, furniture construction, etc. The surfaces of the PU foams are modified by submicrometer polytetrafluoroethylene (PTFE) particles and colloidal superparamagnetic iron oxide nanoparticles (NPs) (Supporting Information, Figure S1 and Figure S2), the latter covering all the reticulated network of the cells inside the volume of the foams. The unmodified foams exhibit inherent hydro- and oleophobicity, whereas when they get modified, they become superhydrophobic, superoleophilic, and magnetic, exhibiting water-repellent, oil-absorbing, and magnetically responsive behavior. Most specifically, the PTFE coating on the foams' surfaces, generated by triboelectric particle deposition, ${ }^{20}$ renders the foams self-cleaning ${ }^{21-23}$ and oil-absorbing. The combination of PTFE particles with colloidal magnetic NPs as foams' coating and the simultaneous loading of the foams with the NPs result in a remarkable increase in the rate of oil absorption. Finally, the imparted magnetic response, due to the superparamagnetic NPs, enables the foams to be magnetically actuated. Such multifunctional foams can easily float on water and be driven by a weak MF to target specific oilpolluted water zones. Given that the PU foam is readily available and that, as we demonstrate next, the NPs can be easily recycled and reused, the proposed application can be scaled up for fast and selective cleanup of large areas of oil spills or for industrial water purification.
The triboelectric PTFE particle deposition is achieved by spreading on the foam's surface PTFE powder, roughly of the same weight as the one of the foam, using a metallic palette, and subsequently rubbing it with the palette against the foam's surface several times. The introduction of oleic acid (OLAC)-capped spherical superparamagnetic iron oxide NPs is done by gently placing the PU foam on a glass substrate where an amount of toluene-based NP solution, containing $12 \%$ of iron oxide with respect to the weight of the bare foam, had previously been casted. The casted solution is totally absorbed by the foam transferring the NPs throughout the entire thickness of the reticulated network of the foam's pores by capillary action. The dimensions of the used foams reassure that the NPs evenly cover the surface of the foams' pores throughout their volume. Many recent studies are focusing on the functional properties that polymer matrices gain after the incorporation in their bulk with magnetic NPs. ${ }^{24-26}$ In these studies, the nanoinclusions were well bound into the polymer, whereas in the present case, the deposited NPs interact with the foams only with weak attractive surface forces. These forces are strong enough to keep the NPs fixed in the foams during the oil-absorption and oil-water separation experiments but weak enough to allow the NPs' removal and recycle after their use as will be described later. On the other hand, the PTFE particle addition onto the foams' surfaces done by contact electrification upon rubbing is forming a stable coating since PTFE when charged triboelectrically can acquire strong electrostatic forces. ${ }^{20}$ Four different types of modified samples were prepared: PU/PTFE, PU/PTFE/NPs, PU/NPs, and PU/NPs/PTFE, with the sequence of the writing of the different components denoting the 
sequence of the application of the respective additives to the pure PU foams.

In Figure 1a-e, the surface morphology of the foam samples modified using combinations of PTFE and colloidal magnetic NP solution is shown. The dimensions of the pores of all the used foams are on the order of a few hundreds of micrometers (Supporting Information, Figure S3), which match with the sizes of recently developed functional meshes for water-oil separation. ${ }^{2,5}$

The pristine PU foam in Figure 1a shows the smoothest surface in comparison with the treated samples, which show increased roughness due to the applied surface modifications. In particular, the surface of the PU/NPs samples (Figure 1b) appears like a crystalline homogeneous layer, while at higher magnification, detailed nanoscale roughness features become visible. The PU/PTFE and PU/NPs/PTFE samples (Figure 1c,d) present aggregated structures on their surface due to agglomerates of PTFE particles, whereas these agglomerates seem to be covered by colloidal NPs in the PU/ PTFE/NPs samples (Figure 1e). The PTFE agglomerates form both micrometer and submicrometer surface roughness features. When the NPs are deposited over the PTFE coating, the presence of hierarchical roughness features at the micrometer, submicrometer, and nanometer scale becomes visible (Figure 1e).

This difference in the surface morphology and chemistry is reflected in the wetting properties of the samples when in contact with water. Specifically, apparent water contact angle (WCA) and WCA hysteresis measurements are shown in Figure 1f. The addition of NPs to the pristine PU foams increases their surface hydrophobicity, and this can be attributed to the induced roughness by the hydrophobic colloidal NPs (WCA on flat NP layers: $101.17 \pm 2.77^{\circ}$ ). However, water drops strongly adhere on their surfaces like in the case of the untreated PU foams. The hydrophobic character of the system remarkably increases in the PU/PTFE and PU/NPs/PTFE samples, where PTFE particles are deposited as the outer layer, rendering them superhydrophobic and self-cleaning, with WCAs greater than $160^{\circ}$ and hysteresis $7.23 \pm 2.31$ and $5.53 \pm 2.21^{\circ}$, respectively. It is well-known that PTFE is a hydrophobic polymer, which can become self-cleaning if some roughness is introduced in its surface morphology. 2,3,22,23,27 In agreement with these findings, the previously shown formation of surface features both at the micrometer and submicrometer scale after PTFE application contributes to the achievement of self-cleaning foam surfaces. Finally, the PU/PTFE/NPs samples are also found to be superhydrophobic, but with diminished self-cleaning character, since the hysteresis is higher compared to that of the PU/PTFE and $\mathrm{PU} / \mathrm{NPs} / \mathrm{PTFE}$ samples. This result is in perfect agreement with a recent publication by Teisala et al. ${ }^{28}$ on superhydrophobic surfaces with hierarchical roughness, which demonstrates that when nanoroughness is introduced water adheres to the surface by penetrating into the nanofeatures.

When instead the foams get modified by magnetic NP solutions with excess free OLAC (f-OLAC), they lose their superhydrophobic character, with the only samples that retain this property being the ones with the exposed PTFE particles. The change in the surface chemistry of the foams due to excess f-OLAC causes both a decrease of WCA and a significant increase of the WCA hysteresis. The role of $\mathrm{f}$-OLAC in the change of the surface properties clearly indicates that when colloidal nanoparticles are used for the modification of the foams the amount of their free capping molecules should be minimized. An analytical discussion on this effect is presented in the Supporting Information.

Oil-wetting properties of the foams were investigated next, by analyzing the behavior of oil drops placed on their surfaces (Figure 1g). The pure PU foams reveal a slightly oleophobic character, with an oil contact angle (OCA) of $86.26 \pm 8.72^{\circ}$. On the contrary, all of the modified foams demonstrate an oil-absorbing character, as the drops deposited on them are rapidly absorbed. This is attributed to the oleophilic character of both the PTFE polymer ${ }^{2,3}$ and the colloidal NPs that drive oil into the foam. Indeed, the OCA of pure NP film was $24.41 \pm 2.19^{\circ}$. Figure $1 \mathrm{~g}$ demonstrates a progressive enhancement in the oil-absorption rate (OAR) of the PU/NPs, PU/PTFE, PU/NPs/PTFE, and PU/PTFE/NPs samples, in the specific order, with the last one being more than 12 times faster than the first one.

Although the PU/PTFE/NPs samples show a superhydrophobic character and the highest OAR, possibly due to the additional nanoroughness introduced by exposed NPs (which can increase the oleophilic character of the system), ${ }^{1,9,29}$ their surface cannot be properly defined as self-cleaning, due to its fairly high WCA hysteresis $\left(10.03 \pm 2.45^{\circ}\right)$. On the contrary, the PU/NPs/PTFE samples, although characterized by a lower OAR, are superhydrophobic with very low hysteresis and can be considered more suitable for cleaning water from oil contaminants since they are expected to move with minimum resistance on the water interface under the application of an external MF. Examples demonstrating the suitability of PU/NPs/ PTFE samples for water/oil separation, due to their extreme water repellency and efficient oil absorbance, are shown in Figure 2a,b.

Figure $2 \mathrm{a}$ shows that an impacting water column ejected from a pipet bounces off the sample, leaving it completely dry. Moreover, when a mixed oil and water drop is placed onto the foam's surface, the oil is rapidly absorbed by the foam, leaving only clean water on its surface (Figure $2 b(1-4)$ ). Since the surface energy of the oil used in this study is $30 \mathrm{mN} / \mathrm{m}^{30}$ close to this of the exposed PTFE layer $\left(\sim 18 \mathrm{mN} / \mathrm{m}^{31}\right)$, the surface of 


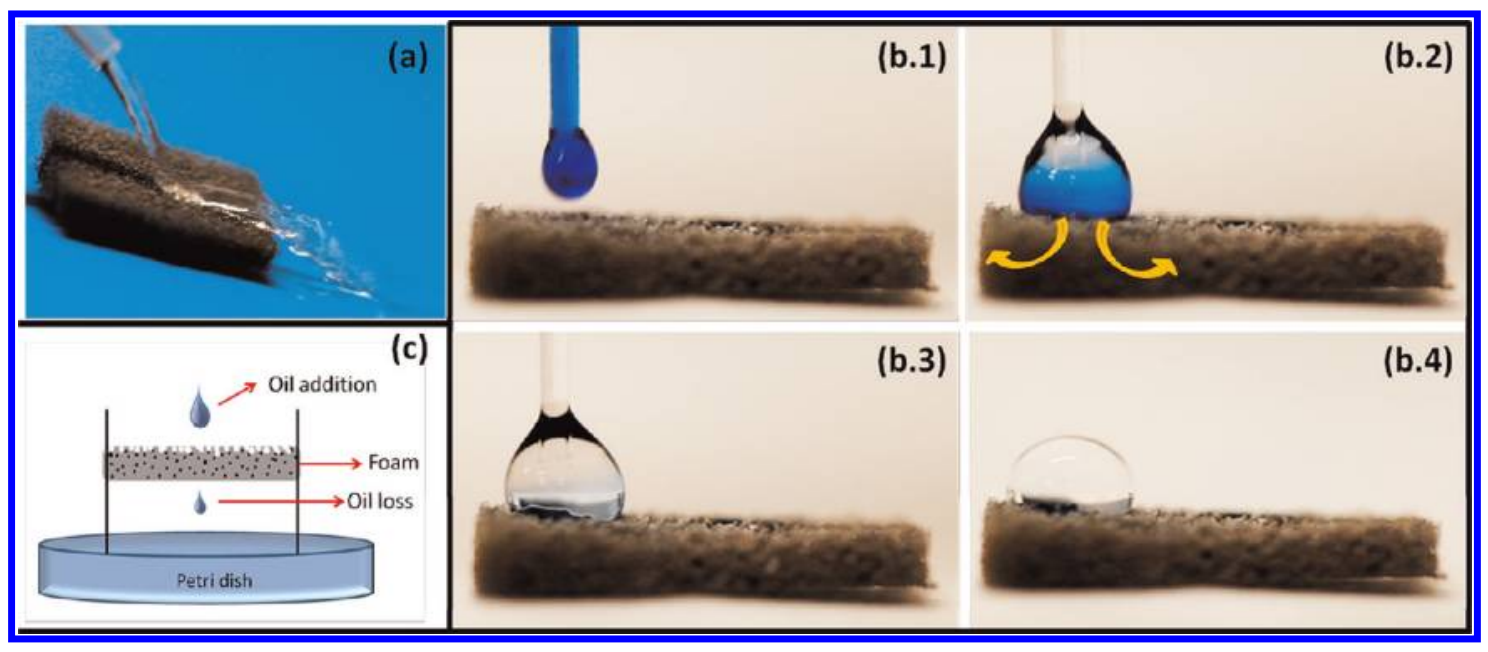

Figure 2. (a) Prolonged water squirt on the PU/NPs/PTFE sample. (b1,2,3,4) Mixed oil (colored with blue dye) and water drop are phase separated, and the oil is immediately absorbed while water remains on the surface. The arrows in (b2) represent the absorption of the oil. Time interval between frames b2 and b3 is less than $1 \mathrm{~s}$. (c) Experimental setup used to determine the foams' oil-absorption capacity. The sample is kept in horizontal position by two needles, and the excess of oil lost through the foam is collected.

the foam is spontaneously wetted by the oil. In contrast, water, due to its high surface tension $72 \mathrm{mN} / \mathrm{m}^{32}$ is strongly repelled by the modified foam's surface. The submicrometer roughness of the exposed layer, attributed to the dimensions of the PTFE particles, enhances these properties, resulting into a superoleophilic and superhydrophobic surface. ${ }^{2}$ The oil is subsequently driven through the porous surface of the foam into its bulk, where its capillary flow is further facilitated due to the oleophilic colloidal NPs decorating the walls of the foam's pores. Since at the same time water stays exclusively at the foam's exterior, the modified foams successfully separate oil from water.

The oil-absorption capacity $c$ of the samples is given by the ratio between the maximum absorbed oil quantity into the foams' volume, $m_{\max }$ and the foams' mass, $m_{\mathrm{f}}\left(c=m_{\max } / m_{\mathrm{f}}\right)$. In order to define $c$ for the PU/ NPs/PTFE foams, mineral oil drops were slowly deposited on a foam sample placed in horizontal position (Figure 2c). The foam absorbs all of the deposited oil up to the quantity $m_{\max }$ above which any additional droplet penetrates through the foam's volume and falls into a collecting pan underneath. Using this method, the capacity was calculated to be $c_{1}=13.25 \pm$ 1.63 , indicating that the maximum weight of oil that the foam can absorb is more than 13 times its weight.

Besides their ideal wetting properties and high oilabsorption capacity, the PU/NPs/PTFE foams can also be magnetically actuated in order to be driven with minimum friction onto polluted water zones by means of a weak MF. In Figure 3a-e are shown photographs of a modified foam floating over a water bath containing the maximum quantity of oil that the foam can absorb, calculated as mentioned above $\left(m_{\max }=\right.$ $13.25 m_{\mathrm{f}}=650 \pm 0.1 \mathrm{mg}$ ).
The foam is moved by a weak MF, toward the oilpolluted zone. Its weight change was measured at different time intervals after it reaches the oil spill, and as shown in Figure $3 \mathrm{f}$, in about $6 \mathrm{~s}$, the weight of the foam increases, reaching almost its final value $\left(m_{2}=\right.$ $601 \pm 0.1 \mathrm{mg}$ ). At this point, since the oil-absorption capacity of the foam is not overcome, no evidence of NPs is noticed on the water surface. The oil-absorption capacity of the foam in this configuration $\left(c_{2}\right)$ is $92 \%$ of the one measured with the configuration presented in Figure $2 c\left(c_{1}\right)$. Anyhow, also in this "more realistic" case, the amount of oil that the foam can absorb is more than 12 times its weight $\left(c_{2}=c_{1} \times 0.92\right)$. It should be mentioned that the measured capacity is similar also when acidic, basic, or seawater are used. The comparison with other polymer-based porous systems reported for oil-water separation reveals that our system presents one of the highest oil-absorption capacities. In fact, although the oil-absorption capacity of the presented foams is still lower compared to that of CNT foams ${ }^{16}$ or polyelectrolyte gels, ${ }^{33}$ it is among the highest values reported for polymeric porous materials. To the best of our knowledge, few studies calculated oilabsorption capacities comparable to the one reported here, ${ }^{10,34}$ whereas others found the weight of the absorbed oil, of similar density as the one used herein, to be $2.92,{ }^{12} 4,{ }^{19} 1.25,{ }^{17}$ and $2^{18}$ times higher than the weight of their initial, dry material, all values well below the one found herein.

As above-mentioned, after use, the NPs can be removed from the oil-containing foams and recycled. In fact, the NPs are detached from the foams' walls and removed together with the absorbed oil after sonication in toluene for a few minutes. In this way, it is possible to separate the NPs from the PU foams and subsequently collect them using a magnet, in order to 


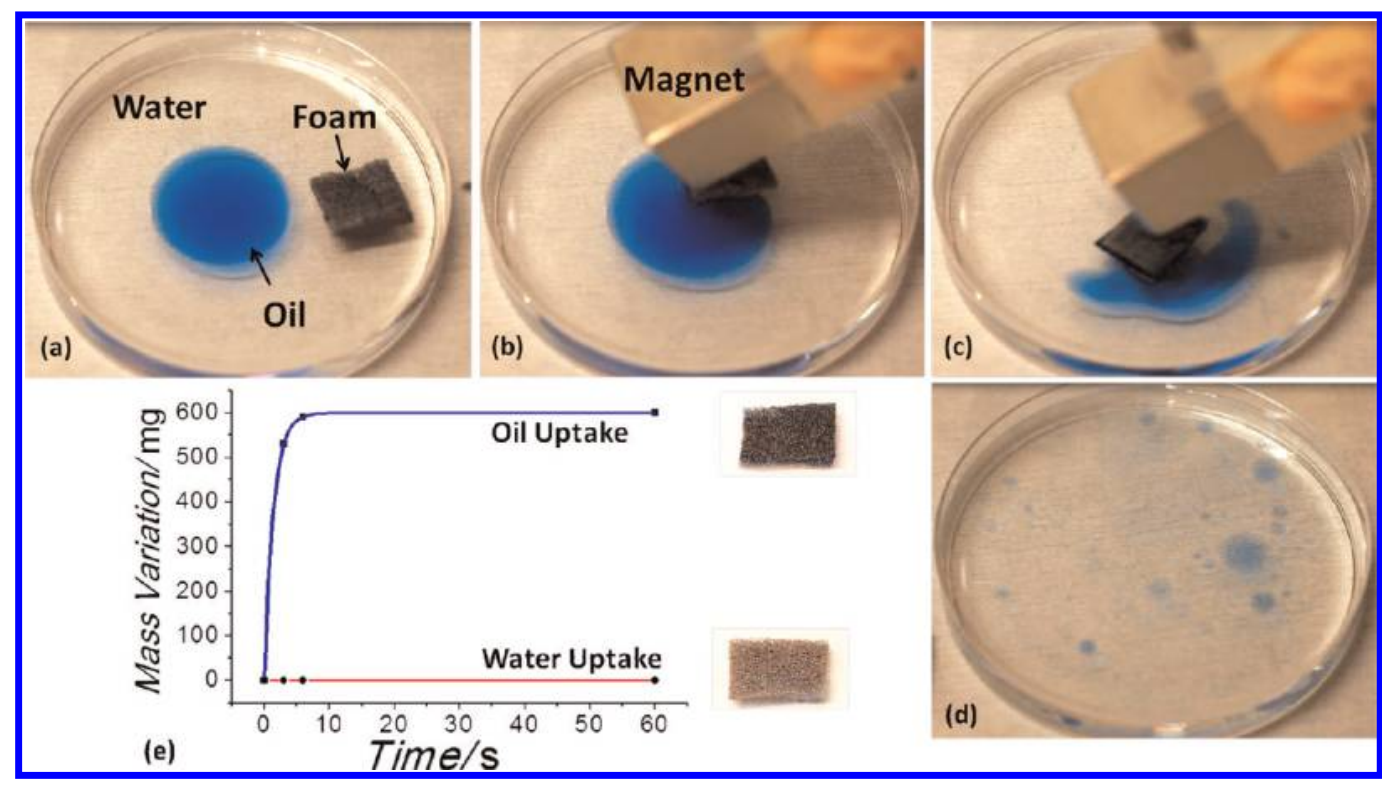

Figure 3. (a) Dye-colored oil on the surface of the water in a Petri dish. (b,c) Subsequent removal of the oil by the magnetically driven PU/NPs/PTFE sample. (d) Cleaned water surface after the process. (e) Graph showing the variation of the weight of the foam with time.

be used again. The quantity of the extracted NPs, and hence the NP extraction efficiency, depends on the weight ratio between the polluted foam and the solvent, toluene. The maximum amount of the NPs that can be recycled is $80 \%$ of the initial quantity based on elemental analysis measurements, and this was obtained for a weight ratio, polluted foam/toluene, of $2 / 145$. Part of the remaining $20 \%$ of NPs is retained in the bulk of the foam, whereas some NPs can also get lost during the magnetic collection.

\section{CONCLUSIONS}

In the present study, a new functional composite material is developed and its water/oil separation properties are demonstrated. In particular, commercially available PU foams were functionalized by means of OLAC-capped superparamagnetic iron oxide
NPs and PTFE particles. From our analysis, it can be concluded that the samples obtained by depositing first the NP solution and then PTFE particles are the most effective in separating oil from water. In fact, such functionalized foams present three important and desirable features: water-repellency, fast oil absorption, and magnetic actuation, attributed to both chemical and morphological features. The fabrication technique is simple and easy to be scaled up, while the employed materials are inexpensive and some of them can be recycled. Therefore, we propose the use of the developed systems in the selective removal of large oil spills from water by means of a magnetic field that can drive the superparamagnetic, waterrepellent, and oil-absorbing foams from a distance toward the polluted areas with minimal energy consumption.

\section{EXPERIMENTAL SECTION}

Materials. The PU foam used as basic material for further modification was donated by VITEC Inc. (U.K.). PTFE particles (diameter 150-200 nm) and all solvents were purchased from Sigma-Aldrich. Spherical NPs of spinel-cubic $\gamma-\mathrm{Fe}_{2} \mathrm{O}_{3} / \mathrm{Fe}_{3} \mathrm{O}_{4}$ were synthesized by a modified surfactant-assisted nonaqueous synthetic approach. ${ }^{35,36}$ In brief, iron pentacarbonyl $\left(\mathrm{Fe}(\mathrm{CO})_{5}\right.$, Aldrich 97\%) was decomposed in hot noncoordinating 1-octadecene solvent (ODE, Aldrich 90\%) in the presence of oleic acid (OLAC, Aldrich 90\%), which has a double role (acting as both oxygen source and as capping agent), at $320^{\circ} \mathrm{C}$ under nitrogen atmosphere for $3 \mathrm{~h}$. Instead of introducing a mild oxidizer in the high-temperature reaction step, the reaction mixture was exposed to air at $120{ }^{\circ} \mathrm{C}$ and annealed for an additional $2 \mathrm{~h}$ to allow oxidation to go to completion. ${ }^{37}$ For the purpose of this work, monodispersed nearly spherical NPs of $10 \pm 1 \mathrm{~nm}$ (see Figure S1 in Supporting Information) were achieved by adjusting the concentration of the precursor $(0.25 \mathrm{M})$ and the surfactant-to-precursor molar ratio (4/1). After allowing the crude reaction mixture to cool to room temperature, the NPs were precipitated by adding 2-propanol, washed several times with acetone to remove precursor residuals and unbound surfactants, and collected by centrifugation. Finally, the precipitate was dissolved in a small amount of toluene. The NPs mainly retain an oleate ligand cap bound to their surface, although the presence of ODE cannot be excluded. ${ }^{24}$ In the present study, two different solutions of colloidal NPs were used. The first one, characterized by a concentration of $170.11 \mathrm{mM}$ (expressed in terms of $\mathrm{Fe}_{2} \mathrm{O}_{3}$ molecular units), contained NPs that have been washed four times, while the second one had a concentration of $75.69 \mathrm{mM}$ and was prepared using NPs that have been washed only once, in order to obtain solutions with different amounts of free (unbound) OLAC and surface-bound oleate anions.

Modification of the Foams. Pieces of PU foams with dimensions of $2.0 \times 1.5 \times 0.2 \mathrm{~cm}^{3}$ (length, width, thickness) were first 
washed by dipping in isopropyl alcohol three times, $10 \mathrm{~min}$ each time, and dried carefully under nitrogen flow. The deposition of PTFE particles exclusively on the surface of the foams was done by triboelectric charging. In particular, triboelectric PTFE particle deposition was achieved by initially spreading a known quantity of PTFE powder on the surfaces of the foams using a metallic palette and subsequently rubbing the metal against the foam surface several times. The deposition of magnetic NPs was done by casting the toluene-based NP solutions onto a glass substrate and then gently placing the thin PU foams on it. The NP solution was transferred throughout the entire thickness of the reticulated network of the foams' pores by capillary action. The dimensions of the used foams reassure that the NPs evenly cover the surface of the foams' pores throughout their volume.

Four different types of modified samples were prepared: (1. PU/PTFE, 2. PU/PTFE/NPs, 3. PU/NPs, 4. PU/NPs/PTFE), with the sequence of the writing of the different components denoting the sequence of the application of the respective additives to the pure PU foams. In particular, in the PU/PTFE samples, only PTFE particles were applied onto the surface of the clean foam, at a relative weight percentage of about $100 \%$ with respect to the weight of the foam; in the PU/PTFE/NPs samples, a toluene solution of colloidal NPs was deposited over the previously deposited PTFE coating, with the relative iron oxide weight percentage being $\sim 12 \%$ with respect to the weight of the foam; in the PU/NPs samples, a toluene solution of colloidal NPs was transferred to the as-received foam, with a relative iron oxide weight percentage of $12 \%$ with respect to the weight of the foam; and finally, in the PU/NPs/PTFE samples, PTFE particles were transferred on the PU foam previously modified by magnetic NPs at a relative weight percentage of about $100 \%$ with respect to the weight of the pure foam. In the case that PU samples were modified using the NP solution with excess free OLAC molecules, we use the notation NPs* instead of NPs.

Characterization of the Colloidal NPs. Magnetic measurements were performed on dried nanocrystal powder samples using a SQUID magnetometer (Quantum Design). Zero-field-cooled (ZFC) magnetization curves were measured by cooling samples in a zero magnetic field and then by increasing the temperature in an applied field of 50 Oe. Field-cooled (FC) magnetization curves were recorded by cooling the samples in a magnetic field of 50 Oe. Magnetization curves were measured by applying a magnetic field up to $50 \mathrm{KOe}$. The results were normalized to the iron oxide mass in the samples (Supporting Information, Figure S2).

The amount of free OLAC/bound oleate in the two colloidal NP solutions was determined by thermogravimetric analysis (TGA), performed using a Mettler Toledo TGA/DSC1 STAR ${ }^{\mathrm{e}}$ System. Samples of $70 \mu \mathrm{L}$, corresponding to about $8 \mathrm{mg}$ of capped iron oxide, from the two different solutions were placed in a platinum pan and analyzed under nitrogen atmosphere. They were first subjected to a heat ramp from 50 to $110^{\circ} \mathrm{C}$ with a rate of $10^{\circ} \mathrm{C} / \mathrm{min}$, and to a final isothermal step for $30 \mathrm{~min}$, in order to evaporate the solvent. They were then heated from 50 to $600{ }^{\circ} \mathrm{C}$ through a temperature ramp of $5^{\circ} \mathrm{C} / \mathrm{min}$ and finally subjected to an isothermal treatment at $600{ }^{\circ} \mathrm{C}$ for $60 \mathrm{~min}$ in order to determine changes in weight related to changes in temperature. The derivative of the weight loss curve was calculated and used to check the inflection points, where the weight losses were most apparent. Following this method, the two NP solutions used were proven to have different OLAC content due to variable degree of purification. Specifically, the first class of NP solution contains $15.1 \mathrm{wt} \%$ of OLAC, while the second one contains $77.5 \mathrm{wt} \%$ of OLAC with respect to the dry weight of NPs (Figure S4 in Supporting Information).

Wetting Properties. Measurements of water and oil contact angles (WCA and OCA, respectively), water contact angle hysteresis, and oil-absorption rate (OAR) were carried out with a KSVCAM200 (Kruss, Germany) contact angle goniometer. Distilled water droplets of $1 \mu \mathrm{L}$ and mineral oil droplets of $4 \mu \mathrm{L}$ were used for measuring the WCA and OCA, respectively. The average contact angle values with corresponding standard deviations were reported for each sample based on 5-8 measurements. For the measurement of the water contact angle hysteresis, advancing and receding contact angles were generated maintaining the sample surfaces at a flat position by increasing and decreasing drop volumes (from 4 to $7 \mu \mathrm{L}$ and vice versa) at the syringe tip. ${ }^{6}$ The difference was recorded as the hysteresis.

The OAR was calculated by recording the variation in time (one frame every $33 \mathrm{~ms}$ ) of the volume of oil droplets deposited onto the surfaces of the samples.

Electron Microscopy Studies. The size and shape of the assynthesized colloidal iron oxide NPs was routinely checked by transmission electron microscopy (TEM) using a JEOL JEM 1011 microscope operating at $100 \mathrm{kV}$. The samples were prepared by dropping a few drops of a dilute NP solution onto 400-mesh carbon-coated copper grids and allowing the solvent to evaporate. Scanning electron microscopy (SEM) images of the samples were recorded using a FEI NOVA NANOSEM200 instrument, in low-vacuum and high-resolution acquisition mode, using a Helix detector. The applied voltage was between 5 and $18 \mathrm{kV}$, under pressure of $1.2 \mathrm{mbar}$.

Water-0il Separation Experiment. The demonstration of the feasibility of cleaning an oil-contaminated water surface by means of the functionalized PU foam was carried out using distilled water and mineral oil (Sigma-Aldrich, viscosity 35.7 cSt, density $0.84 \mathrm{gr} / \mathrm{mL}$ ) colored by Oil Blue 35 dye (Sigma-Aldrich). The magnetic field of the permanent magnet at the operating distance $(3 \mathrm{~cm}$ ) was measured by means of a gaussmeter (Hirst Magnetic Instrumentation Ltd., G.B.) and resulted to be $38 \mathrm{mT}$.

Elemental Analysis. Determination of the concentration of iron in the NP solutions and of the amount of NPs which can be recycled after using the functionalized foam to collect mineral oil pollutant from the water surface was carried out by elemental analysis using an ICP-OES spectrometer (700 Series, Agilent Technologies).

Conflict of Interest: The authors declare no competing financial interest

Acknowledgment. The authors thank Lara Marini of Istituto Italiano di Tecnologia (IIT) Genova, Italy, and Mariada Malvindi of Center for Biomolecular Nanotechnologies (CBN) @UNILE, Istituto Italiano di Tecnologia (IIT), Lecce, Italy, for their support in the performance and interpretation of the TGA analysis and elemental analysis, respectively. The authors thank also Dr. F. Albertini, Dr. V. Chiesi, and Dr. F. Casoli (IMEM-CNR Institute, Parma, Italy) for performing magnetic measurements on the samples.

Supporting Information Available: A TEM image showing the size of the employed colloidal iron oxide NPs; ZFC-FC and magnetic hysteresis figure, showing the magnetic properties of the used NPs; SEM images of the used PU foams; a paragraph where is discussed how the concentration of OLAC into the NP solutions affect the properties of the modified foams; TGA graphs of the two colloidal solutions of iron oxide NPs used; two graphs about the oil and water wettability behavior of the modified foams using NP solution with OLAC in excess; SEM images of the modified foams using NP solution with OLAC in excess. This material is available free of charge via the Internet at http://pubs.acs.org

\section{REFERENCES AND NOTES}

1. Yao, X.; Song, Y.; Jiang, L. Applications of Bio-Inspired Special Wettable Surfaces. Adv. Mater. 2011, 23, 719-734.

2. Feng, L.; Zhang, Z.; Mai, Z.; Ma, Y.; Liu, B.; Jiang, L.; Zhu, D. A Super-Hydrophobic and Super-Oleophilic Coating Mesh Film for the Separation of Oil and Water. Angew. Chem. Int. Ed. 2004, 43, 2012-2014.

3. Feng, X. J.; Jiang, L. Design and Creation of Superwetting/ Antiwetting Surfaces. Adv. Mater. 2006, 18, 3063-3078.

4. Su, C. Highly Hydrophobic and Oleophilic Foam for Selective Absorption. Appl. Surf. Sci. 2009, 256, 1413-1418.

5. Wang, C.; Yao, T.; Wu, J.; Ma, C.; Fan, Z.; Wang, Z.; Cheng, Y.; Lin, Q.; Yang, B. Facile Approach in Fabricating Superhydrophobic and Superoleophilic Surface for Water and Oil Mixture Separation. ACS Appl. Mater. Interfaces 2009, 1, 2613-2617.

6. Lee, C. H.; Johnson, N.; Drelich, J.; Yap, Y. K. The Performance of Superhydrophobic and Superoleophilic Carbon 
Nanotube Meshes in Water-Oil Filtration. Carbon 2011, $49,669-676$.

7. Xue, Z.; Wang, S.; Lin, L.; Chen, L.; Liu, M.; Feng, L.; Jiang, L. A Novel Superhydrophilic and Underwater Superoleophobic Hydrogel-Coated Mesh for Oil/Water Separation. $\underline{A d v}$. Mater. 2011, 23, 4270-4273.

8. Wu, J.; Chen, J.; Qasim, K.; Xia, J.; Lei, W. M; Wang, B.-P. A Hierarchical Mesh Film with Superhydrophobic and Superoleophilic Properties for Oil and Water Separation. J. Chem. Technol. Biotechnol. 2012, 87, 427-430.

9. Zhang, J.; Huang, W.; Han, Y. A Composite Polymer Film with Both Superhydrophobicity and Superoleophilicity. Macromol. Rapid Commun. 2006, 27, 804-808.

10. Yuan, J.; Liu, X.; Akbulut, O.; Hu, J.; Suib, S. L.; Kong, J.; Stellacci, F. Superwetting Nanowire Membranes for Selective Absorption. Nat. Nanotechnol. 2008, 3, 332-336.

11. Wang, S.; Li, M.; Lu, Q. Filter Paper with Selective Absorption and Separation of Liquids That Differ in Surface Tension. ACS Appl. Mater. Interfaces 2010, 2, 677-683.

12. Zhang, J.; Seeger, S. Polyester Materials with Superwetting Silicone Nanofilaments for Oil/Water Separation and Selective Oil Absorption. Adv. Funct. Mater. 2011, 21, 46994704.

13. Yang, J.; Zhang, Z.; Xu, X.; Zhu, X.; Men, X.; Zhou, X. Superhydrophilic-Superoleophobic Coatings. J. Mater. Chem. 2012, 22, 2834-2837.

14. Sun, Z. H.; Wang, L. F.; Liu, P. P.; Wang, S. C.; Sun, B.; Jiang, D. Z.; Xiao, F. S. Magnetically Motive Porous Sphere Composite and Its Excellent Properties for the Removal of Pollutants in Water by Adsorption and Desorption Cycles. Adv. Mater. 2006, 18, 1968-1971.

15. Zhu, Q.; Tao, F.; Pan, Q. Fast and Selective Removal of Oils from Water Surface via Highly Hydrophobic Core-Shell $\mathrm{Fe}_{2} \mathrm{O}_{3} @ \mathrm{C}$ Nanoparticles under Magnetic Field. ACS Appl. Mater. Interfaces 2010, 2, 3141-3146.

16. Gui, X.; Wei, J.; Wang, K.; Cao, A.; Zhu, H.; Jia, Y.; Shu, Q.; Wu, D. Carbon Nanotube Sponges. Adv. Mater. 2010, 22, 617621.

17. Gupta, R.; Kulkarni, G. U. Removal of Organic Compounds from Water by Using a Gold Nanoparticle-Poly(dimethylsiloxane) Nanocomposite Foam. ChemSusChem 2011, 4, 737-743.

18. Thanikaivelan, P.; Narayanan, T.; Pradhan, B. K.; Ajayan, P. M. Collagen Based Magnetic Nanocomposites for Oil Removal Applications. Sci. Rep. 2012, 2, 1-7.

19. Choi, S.-J.; Kwon, T.-H.; Im, H.; Moon, D.-I.; Baek, D. J.; Seol, M.-L.; Duarte, J. P.; Choi, Y.-K. A Polydimethylsiloxane (PDMS) Sponge for the Selective Absorption of Oil from Water. ACS Appl. Mater. Interfaces 2011, 3, 4552-4556.

20. Liu, C.; Bard, A. J. Electrostatic Electrochemistry at Insulators. Nat. Mater. 2008, 7, 505-509.

21. Blossey, R. Self-Cleaning Surfaces-Virtual Realities. Nat. Mater. 2003, 2, 301-306.

22. Patankar, N. A. Mimicking the Lotus Effect: Influence of Double Roughness Structures and Slender Pillars. Lanqmuir 2004, 20, 8209-8213.

23. McHale, G.; Shirtcliffe, N. J.; Newton, M. I. Super-Hydrophobic and Super-Wetting Surfaces: Analytical Potential? Analyst 2004, 129, 284-287.

24. Fragouli, D.; Buonsanti, R.; Bertoni, G.; Sangregorio, C.; Innocenti, C.; Falqui, A.; Gatteschi, D.; Cozzoli, P. D.; Athanassiou, A.; Cingolani, R. Dynamical Formation of Spatially Localized Arrays of Aligned Nanowires in Plastic Films with Magnetic Anisotropy. ACS Nano 2010, 4, 1873-1878.

25. Fragouli, D.; Torre, B.; Bertoni, G.; Buonsanti, R.; Cingolani, R.; Athanassiou, A. Formation and Microscopic Investigation of Iron Oxide Aligned Nanowires into Polymeric Nanocomposite Films. Microsc. Res. Tech. 2010, 73, 952-958.

26. Fragouli, D.; Bayer, I. S.; Di Corato, R.; Brescia, R.; Bertoni, G.; Innocenti, C.; Gatteschi, D.; Pellegrino, T.; Cingolani, R.; Athanassiou, A. Superparamagnetic Cellulose Fiber Networks via Nanocomposite Functionalization. J. Mater. Chem. 2012, 22, 1662-1666.

27. Nilsson, M. A.; Daniello, R. J.; Rothstein, J. P. A Novel and Inexpensive Technique for Creating Superhydrophobic
Surfaces Using Teflon and Sandpaper. J. Phys. D: Appl. Phys. 2010, 43, 1-5.

28. Teisala, H.; Tuominen, M.; Aromaa, M.; Stepien, M.; Mäkelä, J. M.; Saarinen, J. J.; Toivakka, M.; Kuusipalo, J. Nanostructures Increase Water Droplet Adhesion on Hierarchically Rough Superhydrophobic Surfaces. Langmuir 2012, 28, 3138-3145.

29. McHale, G.; Shirtcliffe, N. J.; Aqil, S.; Perry, C. C.; Newton, M. I. Topography Driven Spreading. Phys. Rev. Lett. 2004, 93, 036102-036104.

30. Stoebe, T.; Lin, Z.; Hill, R. M.; Ward, M. D.; Davis, H. T. Superspreading of Aqueous Films Containing Trisiloxane Surfactant on Mineral Oil. Langmuir 1997, 13, 7282-7286.

31. Choi, W.; Tuteja, A.; Chhatre, S.; Mabry, J. M.; Cohen, R. E.; McKinley, G. H. Fabrics with Tunable Oleophobicity. Adv. Mater. 2009, 21, 2190-2195.

32. Vázquez, G.; Alvarez, E.; Navaza, J. M. Surface Tension of Alcohol + Water from 20 to $50^{\circ} \mathrm{C}$. J. Chem. Eng. Data 1995, 40, 611-614.

33. Ono, T.; Sugimoto, T.; Shinkai, S.; Sada, K. Lipophilic Polyelectrolyte Gels as Super-Absorbent Polymers for Nonpolar Organic Solvents. Nat. Mater. 2007, 6, 429-433.

34. Zhu, Q.; Pan, Q.; Liu, F. Facile Removal and Collection of Oils from Water Surfaces through Superhydrophobic and Superoleophilic Sponges. J. Phys. Chem. C 2011, 115, 17464-17470.

35. Hyeon, T.; Lee, S. S.; Park, $V_{i}$ Chung, $V_{i}$ Na, H. B. Synthesis of Highly Crystalline and Monodisperse Maghemite Nanocrystallites without a Size-Selection Process. J. Am. Chem. Soc. 2001, 123, 12798-12801.

36. Park, J.; Lee, E.; Hwang, N.-M.; Kang, M.; Kim, S. C.; Hwang, Y.; Park, J.-G.; Noh, H.-J.; Kim, J.-Y.; Park, J.-H.; et al. OneNanometer-Scale Size-Controlled Synthesis of Monodisperse Magnetic Iron Oxide Nanoparticles. Angew. Chem. Int. Ed. 2005, 44, 2872-2877.

37. Cozzoli, P. D.; Snoeck, E.; Garcia, M. A.; Giannini, C.; Guagliardi, A.; Cervellino, A.; Gozzo, F.; Hernando, A.; Achterhold, K.; Ciobanu, N.; et al. Colloidal Synthesis and Characterization of Tetrapod-Shaped Magnetic Nanocrystals. Nano Lett. 2006, 6, 1966-1972. 